\title{
Response to Letter to the Editor from Lui et al.: 'Euthyroid sick syndrome as an early surrogate marker of poor outcome in mild SARS-CoV-2 disease': prognostic significance of non-thyroidal illness syndrome in the whole spectrum of COVID-19 severity
}

\author{
C. Sparano ${ }^{1} \cdot$ M. Maggi ${ }^{1} \cdot$ L. Petrone ${ }^{1,2}($ )
}

Received: 20 December 2021 / Accepted: 21 December 2021 / Published online: 28 January 2022

(C) Italian Society of Endocrinology (SIE) 2021

\section{Dear Editor,}

We carefully read the "Letter to the Editor" by Dr Lui et al. in reply to our study on the role of euthyroid sick syndrome (ESS) in mild COVID-19 patients [1]. The authors questioned the almost ten times higher rate of ESS diagnosed in our population (i.e. $57 \%$ vs $5.2 \%$ for Lui et al.'s mild population [2]) and raised some concerns about the SARS-CoV-2 disease-severity classification applied. In that respect, we would like to point out some criticisms and our interpretation of such divergent results.

At present, several clinical scores have been used to stratify SARS-CoV-2 infection, but none of them have demonstrated superiority over the others. In fact, as summarized in the supplementary appendix of the Hodgson et al. study [3], we can find at least eight different classifications, with only a few having been previously validated. The chosen World Health Organization Ordinal Scale for COVID-19 represents an easy tool to weigh the infection burden, since the intensity of oxygen supply acts as a clinical warning for patients' health deterioration. On the other hand, the parallel Chinese National Health Commission guidelines define as mild disease subjects with general (and non-specific) mild clinical symptoms and no signs of pneumonia at chest imaging, which may fit with the large majority of our cases. Thereafter, the Chinese scale becomes more explicit within the severe disease class, but with broader criteria: for instance,

L. Petrone

luisa.petrone@unifi.it

1 Endocrinology Unit, Department of Experimental and Clinical Biomedical Sciences "Mario Serio", University of Florence, Florence, Italy

2 Endocrinology Unit, Medical-Geriatric Department, Azienda Ospedaliero-Universitaria Careggi, Largo Brambilla 3, 50134 Florence, Italy patients with $\mathrm{PaO}_{2} / \mathrm{FiO}_{2}$ levels from $300 \mathrm{mmHg}$ downward are scored as severe. Nonetheless, if we consider the same parameter, according to the universally accepted "Berlin criteria" for acute respiratory distress definition, for up to $200 \mathrm{mmHg}$ of $\mathrm{PaO}_{2} / \mathrm{FiO}_{2}$ levels, the oxygen impairment is classified as mild, suggesting limited lung damage, which applies to our population. This means that, by adopting the Chinese scale, a large amount of our sample would have been over-scored in terms of disease severity, even without needing intensive care or higher oxygen supports. This point is not totally in contradiction with Lui et al.'s commentary, since we agree that the available COVID-19 classifications fail to provide a satisfying stratification of the infection activity. In line with the above, we are now aware that the first clinical appearance, although significant, is not suited to identify frailer patients. Moreover, Lui et al. [2] considered a very heterogeneous population, including asymptomatic carriers of SARS-CoV-2 infection, i.e. those screened during tracing procedures and hospitalized for clinical monitoring. Conversely, our protocol included only cases that required hospital care or showed initial clinical deterioration, thus implying a mild, but substantial disease activity. Finally, another point to highlight is the larger time span of the pandemic covered by our study, where the differing virulence of different SARS-CoV-2 variants may have played a further role on thyroid axis disfunction.

Based on these premises, in agreement with Lui et al.'s suggestions, we look forward to a more suitable clinical scoring system, able to overcome the present limitations. At the same time, we also hope for a further role of thyroid function in a smarter biochemical panel for COVID-19 disease stratification. 


\section{Declarations}

Conflicts of interest M.M. is an associate of JENI Editorial Board (for the Andrology section). All the other authors have no conflicts of interest and nothing to disclose.

Ethical approval Not applicable.

Informed consent Not applicable.

\section{References}

1. Sparano C, Zago E, Morettini A et al (2021) Euthyroid sick syndrome as an early surrogate marker of poor outcome in mild
SARS-CoV-2 disease. J Endocrinol Invest. https://doi.org/10. 1007/s40618-021-01714-1

2. Lui DTW, Lee CH, Chow WS et al (2021) Role of non-thyroidal illness syndrome in predicting adverse outcomes in COVID-19 patients predominantly of mild-tomoderate severity. Clin Endocrinol (Oxf) 95(3):469-477. https://doi.org/10.1111/cen.14476

3. Hodgson SH, Mansatta K, Mallett G et al (2021) What defines an efficacious COVID-19 vaccine? A review of the challenges assessing the clinical efficacy of vaccines against SARS-CoV-2. Lancet Infect Dis 21:e26-e35. https://doi.org/10.1016/S1473-3099(20) 30773-8

Publisher's Note Springer Nature remains neutral with regard to jurisdictional claims in published maps and institutional affiliations. 